\begin{tabular}{|c|c|c|c|}
\hline Eiszeitalteru. Gegenwart & $\mathbf{2 7}$ & $\begin{array}{c}159-173 \\
4 \mathrm{Abb} ., 3 \mathrm{Tab} .\end{array}$ & Öhringen/Württ. 1976 \\
\hline
\end{tabular}

\title{
Das Jungholozän im Reichenhaller Becken
}

\author{
Karl Brunnacker, Jürgen Freundlich, Manfred Menke \& Hans Schmeidl *)
}

Late glacial terrace, meanders, erosion, fluvial sediments, peat, Bronze Age, fluvial fan, lake, saline springs

K u rzf a s s u n g: Für das jüngere Holozän des Reichenhaller Beckens und der Saalach wird eine Giederung entwickelt, die sich sowohl hinsichtlich der Generaltendenzen als auch der zwischengeschobenen Aktivierungsphasen in das Bild der allgemeinen Flußentwicklung Mitteleuropas einfügt:

1. Froschhamer Terrasse (spätglaziale Rückzugsterrasse)

2. Saalach-Mäander auf der Froschhamer Terrasse

3. Tiefe Saalach-Einschneidung $=$ entsprechend der Aktivierungsphase "Pulling Stufe“ rd. 4300 a BP

4. Feinsedimente mit Torf im Becken (bronzezeitlich)

5. Spätbronzezeitlicher Schwemmkegel $=$ rd. 3000 Jahre vor heute sowie Lokalschotter randlicher Schwemmfächer (anthropogen ausgelöst, z. T. etwas älter)

6. Bereichsweise minerogene Feinsedimente mit Torf im Becken

7. Saalach-Schwemmkegel, randlich am Becken Auelehm = Aktivierungsphase „LerchenfeldStufe" (ab 2. Jh. n. Chr.)

8. Ehemaliger Saalach-See sowie mittelalterliche bis neuzeitliche Saalach-Schotter im Auebereich. Damit ist es im Becken nicht möglich, im Unterschied zu seinen Randhöhen, eine prähistorische Besiedlung zu fassen.

Die Sole-Quellen sind auf den bronzezeitlichen Talboden eingestellt. Die spätere Auffüllung des Beckens hatte auf das Niveau dieser Austritte, etwa über einen Rückstau, keinen Einfluß.

[The Young Holocene in the Reichenhall Basin]

Abstract: In the Reichenhall basin a late glacial terrace is locally developed, which is related to the ice retreat. This terrace is carved by meanders of the early holocene Saalach. The late holocene starts with a phase of strong erosion. Afterwards the basin was filled up with fluvial fine-grained sediments and peat during the Bronce Age. Above, gravels of a late Bronce age fluvial fan are deposited. They are again superposed by fine-grained sediments and peat. Beginning with the $2^{\text {nd }}$ century A. D. a Saalach fluvial fan and marginal loam follow. In the Middle Ages a lake forms the youngest deposits of the basin.

Such an organization of holocene fluvial deposits is comparable as well concerning the general trend as intercalated, short-termed phases of activity with findings in other parts of Middle Europe.

Contrary to the surrounding hills prehistoric settlements are not known inside the basin, due to the sediments which are partially more than $15 \mathrm{~m}$ thick.

The valley floor of the Bronze Age is the level surface of the saline springs of Reichenhall. The subsequent sedimentation didn't influence that level surface.

*) Anschrift der Verfasser: Prof. Dr. K. B r u n n a cker, Geol. Inst., Zülpicher Str. 49, 5000 Köln 1, Dr. J. Fre un d li ch, Inst. f. Ur- u. Frühgesch., Weyertal 125, 5000 Köln 41, Doz. Dr. M. M e nke, Inst. f. Vor- u. Frühgesch., Meiserstr. 6, 8000 München 2, Dr. H. S c h m e id l, Bayer. Landesanst. f. Bodenkultur u. Pflanzenbau, Moorforschungsstelle, 8214 Bernau/Chiemsee. 
Die Nacheiszeit war zwischen Alpen und Nordsee keineswegs die ereignislose Zeit, als die sie bislang angesehen werden durfte. Neben den bekannten Eingriffen des Menschen ab dem Neolithikum deuten neuere Forschungen auf Entwicklungstendenzen wie denselben aufgesetzte, relativ kurzfristige Ereignisse (BRUNNACKer 1974). Ein Beispiel dafür bietet das Reichenhaller Becken. Über seine Sole-Quellen war es seit langem ein wirtschaftlich und siedlungsmäßig bedeutsamer Raum. Doch kann seine heutige Topographie nicht unbegrenzt auf frühere Zeiten übertragen werden.

Abgeschlossen wurden die Untersuchungen im Jahr 1974. Herr F. Hofmann, Bad Reichenhall, hat sie in vielerlei Hinsicht, durch Vermittlung von Bohrdaten, Torfproben (REI 4) sowie eigene Beobachtungen, unterstützt. Dafür danken wir. Der geobotanische Anteil wurde von H. SChmeIDL, der archäologische von M. Menke, die absolute Datierung von J. FreundLich und der geologische Anteil von K. BrunNaCKer bearbeitet.

\section{Geologie und Geomorphologie}

Im Umkreis des Reichenhaller Beckens stehen vorzugsweise Karbonatgesteine mit Höhen bis $1700 \mathrm{~m}$ NN im Norden und Süden an. Die Reliefdepression dazwischen liegt heute bei $470 \mathrm{~m}$ NN. Im Osten und Westen bilden bis $800 \mathrm{~m}$ NN hohe Riegel die Begrenzung (Krauss 1913, v. Hillebrandt 1962, Herm 1962). Die Form des Beckens ist vorzugsweise durch Gletscherexaration bedingt, die bis über $100 \mathrm{~m}$ unter den heutigen Talboden hinabgegriffen hat. Seeton, Seekreide und Schotter, insbesondere der Saalach, bilden die seitherige Verfüllung. Eingeleitet wurde die Verfüllung durch den Eisabbau, der schon vor dem Alleröd eingesetzt hat (Schmeidl 1971, Heuberger 1972).

\section{Oberflächennahe Ablagerungen des Beckens}

Verschiedenalterige Saalach-Schüttungen liegen bei Reichenhall etwa niveaugleich, so daß sie sich nur grob gliedern lassen (Abb.1). Aber immerhin ist es möglich, sie mittels Pollenanalyse, Archäologie und C14-Datierung in ein Zeit-Schema einzufügen (Abb. 2).

Vorbemerkung zur Geobotanik:

Da die Altersdatierung des Holozäns im Bereich des Beckens auf Grund der Ablagerungen allein nicht möglich war, wurde versucht, dieselbe u. a. mit Hilfe der Pollenanalyse zu lösen. Wir gingen davon aus, daß der Polleninhalt der in Kiesen, Sanden und Tonen eingelagerten organischen Substanz - sei es Torf oder toniger Dy - Angaben liefert, die für eine Datierung Hinweise geben. Es war uns aber auch klar, daß die Zuordnung von Einzelproben mit Unsicherheiten behaftet ist und dies im besonderen Maße, solange kein Standard-Diagramm aus dem Reichenhaller Becken vorliegt. Postglaziale paläobotanische Untersuchungen liegen vor: aus dem Leopoldskroner Moor (Firbas 1923), aus dem Ainringer Moor (Paul \& Ruoff 1927), aus der Pechschnait bei Traunstein (Schmeidl unveröff.), vom Frillensee (Nordseite des Staufens aus $900 \mathrm{~m}$ - Schmeidl 1973), aus dem Berchtesgadner Land (MAYER 1966) und aus dem nördl. Salzburger Land (Lürzer 1956). Diese Arbeiten ergaben Anhaltspunkte für die Einordnung der einzelnen Proben in die Waldgeschichte, die in großen Zügen wohl auch für das Reichenhaller Becken Gültigkeit hat. Es werden daher die mit Hilfe der pollenanalytischen Untersuchungen gezogenen geologischen Folgerungen mit großer Wahrscheinlichkeit zutreffend sein.

Die Aufbereitung der Proben erfolgte teils mit Schwerelösung $\left(\mathrm{ZnCl}_{2}\right)$ - nach BEug (schriftlich) - und einer nach Frenzel abgeänderten Methode.

\subsection{Froschhamer Terrasse und deren Deckschichten}

Die Oberfläche dieser am Ausgang des Beckens erfaßten Terrasse liegt in 470 bis 460 m NN. Aufgebaut wird sie aus schlecht sortiertem grobem Geröll mit etwas Zentralalpin und sandigem grauem $Z$ wischenmittel. Anscheinend handelt es sich um einen Terrassenrest aus der Zeit des Eisrückzuges, der zwischen Eisrand und östlichem Rand des 


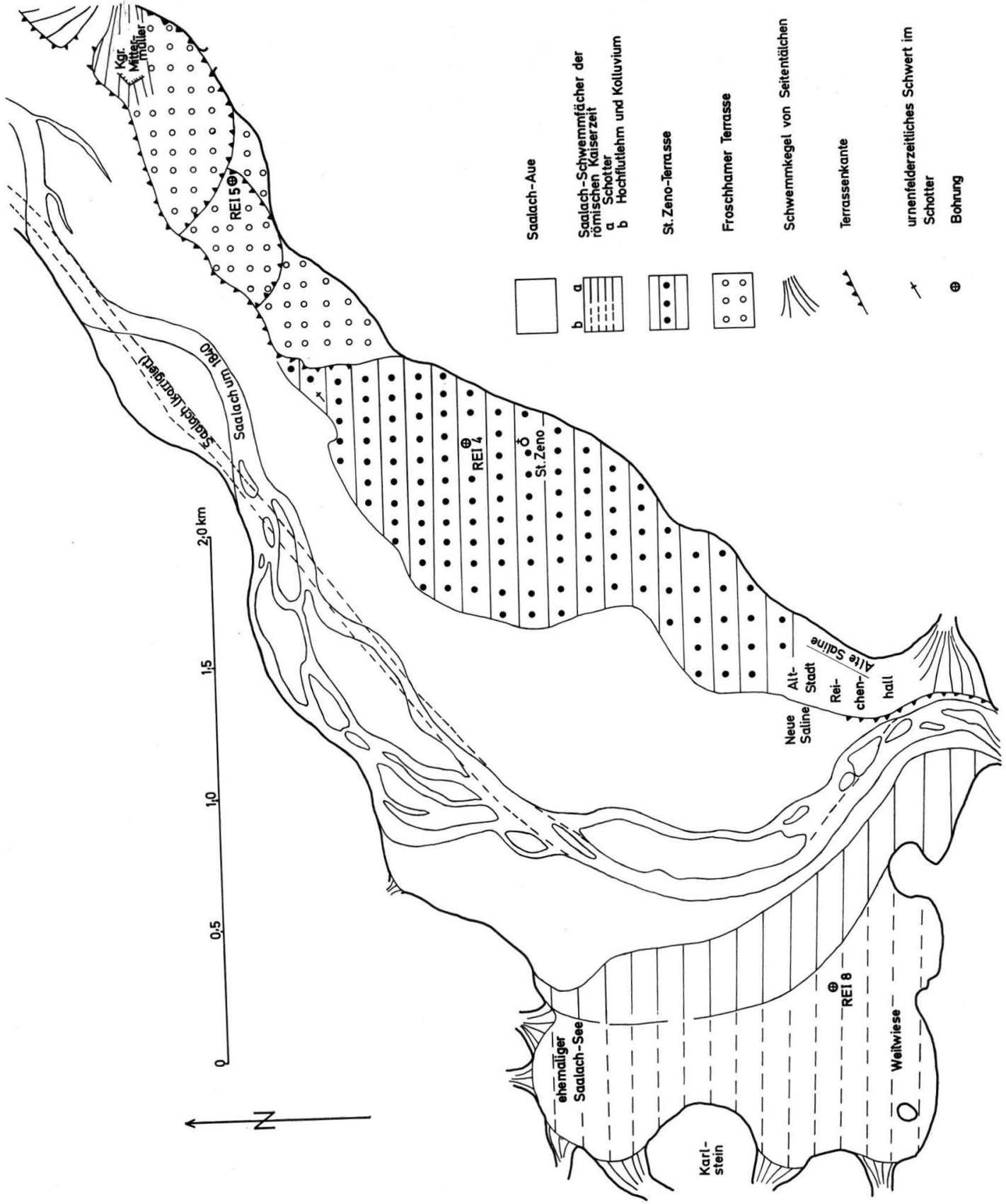

Abb. 1: Das Holozän im Reichenhaller Becken. 
Beckens aufgeschottert wurde. Talabwärts kann die Terrasse bis Marzoll verfolgt werden. Ihre Oberfläche ist durch Mäanderbögen der Saalach gegliedert. Doch haben jüngere Schotterdecken geringer Mächtigkeit (oft nur $1 \mathrm{~m}$ ) diese Formen verwischt. Möglicherweise entsprechen den auflagernden Schwemmfächern auch andernorts junge Ablagerungen in Tälchen, die in das Becken hinausmünden.

$$
\begin{array}{l|l|l|l|l|l}
\text { NE Froschhamer Terrasse } \mid & \text { St.Zeno Terrasse | Saalach-Aue } & \text { SE } & \text { SE }
\end{array}
$$

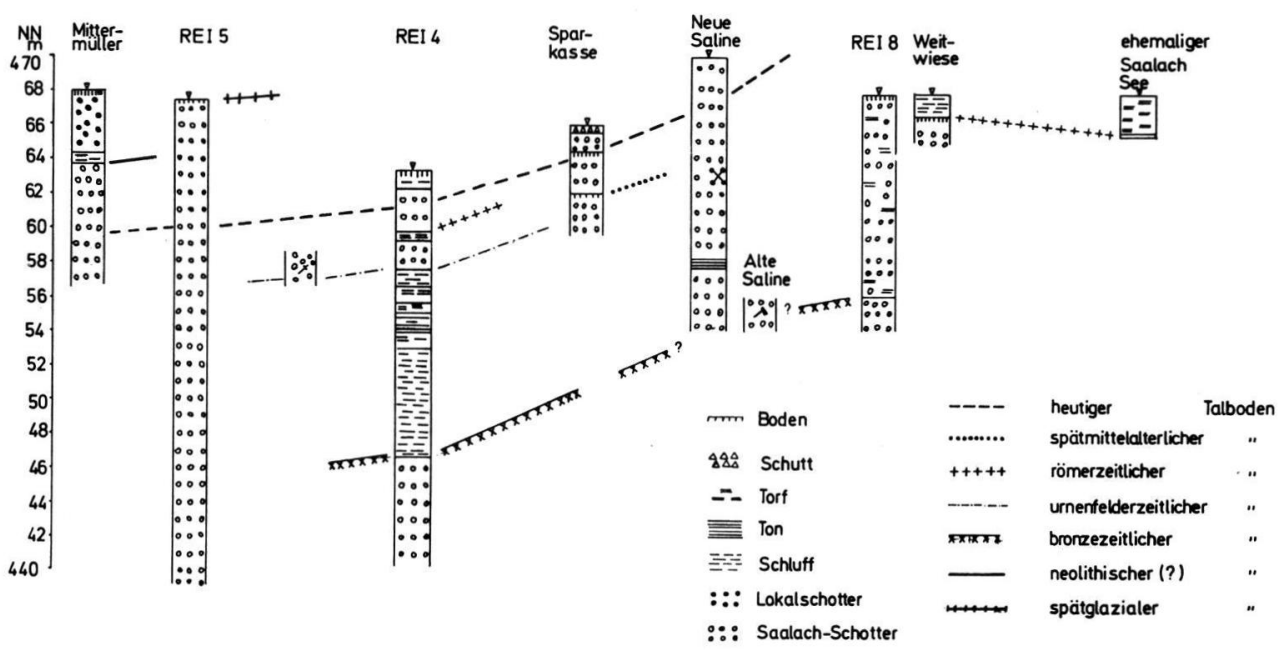

Abb. 2: Aufbau des Holozäns im Reichenhaller Becken nach Bohrungen und Aufschlüssen.

Profil Kiesgrube „Mittermüller" nördlich Bad Reichenhall (462 m NN, Abb. 3):

Probe- Mächtigkeit

\begin{tabular}{|c|c|c|}
\hline 1 & 30 & $\begin{array}{l}\text { Humushaltiger, dunkelgrauer, schwach toniger, sandiger Lehm, bröckelig; } \\
\text { seitlich mit } \mathrm{B}_{\mathrm{V}} \text {-Horizont an der Untergrenze }\end{array}$ \\
\hline 2 & 20 & gelbgrauer, sandiger Kleinkies \\
\hline 3 & bis & schwach humushaltiger, braungrauer, schwach kiesiger, sandiger Lehm \\
\hline 4 & bis 20 & graugelber, schwach sandiger Mittelkies, stark rostfleckig \\
\hline 5 & 100 & gelbgrauer, schwach sandiger Kies \\
\hline 6 & bis 10 & blaugrauer, sehr schwach kiesiger, toniger, sandiger Lehm \\
\hline 7 & 150 & gelbgrauer, schwach sandiger Klein- bis Mittelkies \\
\hline 8 & $\overline{10}$ & schwach humushaltiger, braungrauer, sandiger Lehm \\
\hline 9 & $\begin{array}{l}5 \\
2\end{array}$ & $\begin{array}{l}\text { grauer, lehmiger Sand } \\
\text { torfiger, lehmiger Sand, dunkelgrau }\end{array}$ \\
\hline 10 & 10 & $\begin{array}{l}\text { humushaltiger, dunkelgrauer, lehmiger Sand und sandiger Lehm, mm-dick } \\
\text { gebändert }\end{array}$ \\
\hline 11 & 3 & dunkelgrauer, torfiger, lehmiger Sand \\
\hline $12+13$ & 15 & schwach humushaltiger, braungrauer, sandiger Lehm \\
\hline & 10 & dunkelgraubrauner, schwach torfiger, sandiger Lehm \\
\hline 15 & $\begin{array}{r}12 \\
1\end{array}$ & $\begin{array}{l}\text { graubrauner, toniger, sandiger Lehm } \\
\text { dunkelgrauer, toniger, sandiger Lehm }\end{array}$ \\
\hline 16 & 10 & grauer, toniger, sandiger Lehm, rostfleckig \\
\hline 17 & $\begin{array}{r}-\overline{20} \\
700\end{array}$ & $\begin{array}{l}\text { Geröllkondensat (bis } 15 \mathrm{~cm} \text { Stück- } \phi \text { ) } \\
\text { grauer, sandiger Grobkies (bis } 20 \mathrm{~cm} \phi \text { ), mit Kristallin }\end{array}$ \\
\hline
\end{tabular}


In Nr. 17 ist die Froschhamer Terrasse erfaßt. Die Mäanderbildung wird darüber durch ein Kondensat aus grobem Geröll angezeigt. In Nr. 16 bis 8 folgt eine z. T. humose, sandig-lehmige Füllung mit Torf-Schmitzen. Darauf lagert der Lokalschotter.

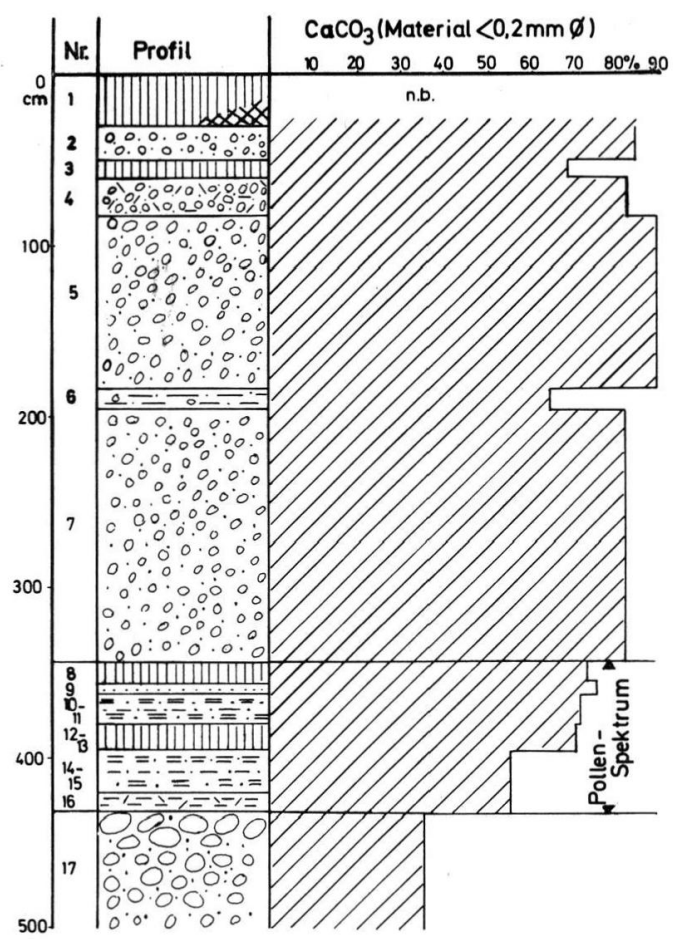

Abb. 3: Profil „Mittermüller", geologischer Aufbau.

Geobotanik Profil „Mittermüller“ (Abb. 4):

Der Erhaltungszustand der Pollen war vereinzelt gut. In Nr. 8, 11 und 13, z. T. waren sie z. B. aber auch stark korrodiert. Das Spektrum in Nr. 15 ist pollenarm.

Die Spektren lassen sich zu einem Diagramm zusammenfassen, in dem die Buche z. T. dominiert. Über das genaue Alter läßt sich keine Aussage machen. Vermutlich gehört aber die unterste Probe noch dem Atlantikum an und das Fagus-Maximum in das Subboreal. Auffallend ist der nur spärlich vertretene Eichenmischwald (EMW). Daß die Spektren mit höheren Buchenwerten gegen das Ende des Subboreal, wenn nicht sogar in das Subatlantikum zu stellen sind, lassen die kulturanzeigenden Pollen vermuten, die im Spektrum 12 schon anwesend sind.

Gemäß diesem Aufschluß hat die Saalach gegen Ausgang des Atlantikum die Tendenz zur Mäanderierung aufgegeben und sich stärker eingeschnitten. Ob bis dahin im Becken ein größerer See bestanden hat, ist anhand der vorerst verfügbaren Daten nicht zu entscheiden. Andernorts lassen die Beckenfüllungen ebenfalls einen komplizierten und wechselvollen Ablag erkennen (z. B. JANIK 1969, BRUNNACKER 1967).

Die Mäanderrinnen wurden seit dem Subboreal mit minerogenem und auch organogenem Material gefüllt. Ein Lokalschotter, der in dem besprochenen Profil bereits in das 


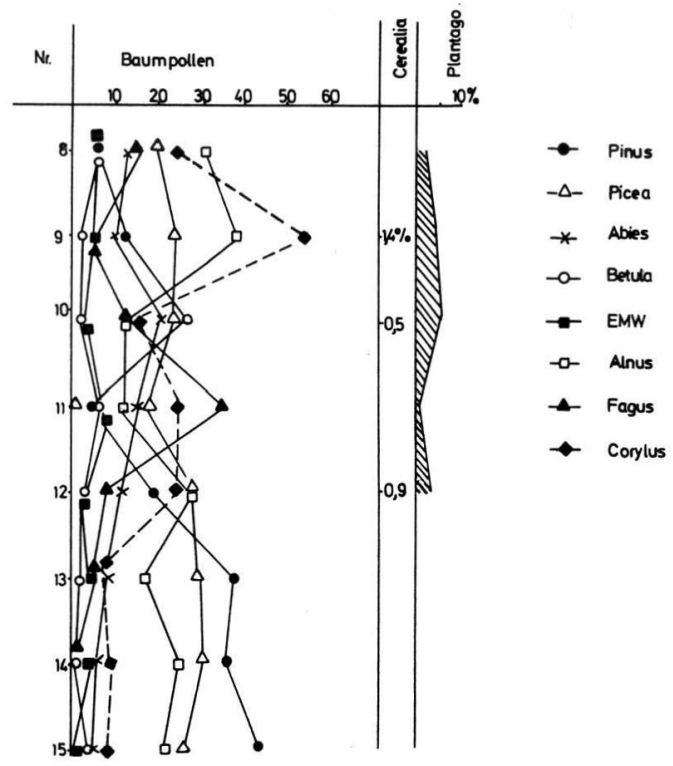

Abb. 4 : Profil „Mittermüller“, Pollendiagramm.

Subatlantikum hinüberleitet, deckt flächig darüber hinausgreifend weite Bereiche am Rand der damaligen Saalach-Aue ein. Ausgelöst wurde die Überlagerung wahrscheinlich durch Störung der Vegetationsdecke infolge Betätigung des Menschen. Demgemäß ist die Abfolge mit vorgeschichtlichen Funden verknüpft. So sind z. B. bei Marzoll $(12 \mathrm{~km}$ nordöstlich des Kartenausschnittes Abb. 1) aus einem solchen Bereich bronzezeitliche Siedlungsspuren und ein urnenfelderzeitliches Gräberfeld, also 3000 bis 3500 Jahre zurück, bekannt geworden.

\subsection{St. Zeno-Terrasse}

Die St. Zeno-Terrasse wurde zwischen heutiger Saalach-Aue und E-Rand des Beckens mit Oberfläche rd. $5 \mathrm{~m}$ unter dem Niveau der Froschhamer Terrasse ausgeschieden. Auf ihrem südlichen Bereich liegt die Altstadt von Reichenhall. Der Aufbau der Terrasse und der in ihr eingeschlossenen archäologischen Funde liefert zur jüngeren Geschichte des Bekkens wesentliche Befunde.

Bohrung „REI 4 (St. Zeno)“:

In der Bohrung REI 4 (Abb. 2) wurden den Schottern zwischengeschobene feinkörnige bis torfige Bildungen durchfahren. Torf liegt in einer Teufe von 3,5-4,2 m. Darunter folgen in $6 \mathrm{~m}$ bis rd. $17 \mathrm{~m}$ Teufe weitere Schluffe und Tone, die mit Torf durchsetzt sind. Das Liegende bildet im wesentlichen bis zur Teufe rd. $50 \mathrm{~m}$ wiederum Schotter. Die zwischengeschalteten, torfigen Tone und Schluffe weisen auf Altwasser- und Hochflutabsätze, also auf eine Zeit erheblich geminderter fluviatiler Aktivität hin.

Geobotanik Profil „REI 4“ (Tab. 1):

Probe 1: $\quad$ Torfige Lage bei $7 \mathrm{~m}$ Teufe

Kalkhaltiges, blättriges Material, mit vielen humosen Einschlüssen: Laubblatt-Fragment von cf. Alnus, Holzreste von cf. Coniferen, Reste von Braunmoos, Laubbaum- 
rinden und Kräutern. Pollenerhaltung war gut. Der EMW ist ganz schwach vertreten, dafür erreicht Fagus $24 \%$. Alnus ist sehr stark vertreten (vermutlich lokal). Von Kulturanzeigern wurden gefunden: Plantago lanc. $2 \%$, Chenopodiaceen 0,5\%, Cruciferen $2 \%$, Compositen $1 \%$, außerdem varia und indeterminata $11 \%$.

Probe 2: Torfige Lage bei $16 \mathrm{~m}$ Teufe

Kalkhaltiges, blättriges Material: Radizellentorf mit Scorpidium. Rinden- und Laubblattreste cf. Alnus.

Aus dem vorhandenen Stück wurden 2 Proben entnommen. Da die Probe nicht orientiert war, kann nicht gesagt werden, welche oben bzw. unten war:

Probe 2a: Die Probe beinhaltet hohe Coniferenwerte, sehr niedrige EMW- und Buchenwerte von $16 \%$. Kulturanzeiger: Plantago $2 \%$, Chenopodiaceen $1 \%$, Compositen $5 \%$.

Probe 2b: In dieser Probe liegt Abies etwas niedriger als in 2a. Der EMW ist ebenfalls mit niedrigen Werten vertreten. Die Buche liegt mit $24 \%$ höher als in der anderen Probe. Als Kulturanzeiger wurden gefunden: Plantago: $1 \%$, Compositen $2 \%$, Cruciferen $1 \%$.

Tab. 1: Pollenspektren in der Bohrung „REI 4“ (Pollenzahl in \%)

\begin{tabular}{|c|c|c|c|}
\hline & $\begin{array}{c}1 \\
(7 \mathrm{~m} \text { Tiefe })\end{array}$ & \multicolumn{2}{|c|}{ (16 m Tiefe) } \\
\hline Pinus & 6 & 14 & \\
\hline Picea & 18 & 18 & 21 \\
\hline Abies & 11 & 15 & \\
\hline Betula & 9 & 15 & 12 \\
\hline Quercus & 5 & 3 & \\
\hline Tilia & - & 1 & \\
\hline Ulmus & 2 & - & \\
\hline Fraxinus & 1 & - & \\
\hline EMW & 8 & 4 & \\
\hline Alnus & 24 & 18 & 20 \\
\hline Fagus & 24 & 16 & \\
\hline Carpinus & 0,5 & - & \\
\hline Salix & - & 1 & - \\
\hline Corylus & 38 & 29 & \\
\hline Gramineen & 20 & 5 & 12 \\
\hline Cyperaceen & 15 & 10 & \\
\hline Plantago & 2 & 2 & \\
\hline Compositen & 1 & 5 & \\
\hline Chenopodiaceen & 0,5 & 1 & \\
\hline
\end{tabular}

Die Ablagerung des torfigen Materials in $16 \mathrm{~m}$ Teufe erfolgte in der Buchenzeit, und zwar nach dem Abfall der EMW-Kurve. Auf Grund von C14-Datierungen liegt der Schnittpunkt von EMW zu Fagus im oberbayerischen Raum bei rd. 2500 v. Chr. In den südlichen Chiemsee-Mooren wurde das Alter dieses Diagramm-Abschnittes mit $4750 \pm 50$ a BP und im Gebiet des Starnberger Sees mit $4440 \pm 70$ a BP (SchmeIDL, unveröffentlicht) bestimmt, was dem oben genannten Alter entspricht. Damit wäre die Ablagerung in $16 \mathrm{~m}$ Tiefe jünger.

Bohrung „REI 8 “ und „Pfahlgründung Neue Saline“:

In das sich herausschälende Bild über das jüngere Holozän fügen sich frühere Beobachtungen beim Kanalbau nördlich des Elektrizitäts-Werkes (KRAUss 1913) und Angaben über die Bohrung REI 8 ein. Demnach sind dort die obersten Bereiche des Schotters mit Torf und z. T. mit Seekreide durchsetzt. In REI 8 geht diese Fazies bis $12 \mathrm{~m}$ Teufe hinab. 
Hinzu kommt ein Pferdeknochen im neuen Salinengelände in $7 \mathrm{~m}$ Teufe. In $12 \mathrm{~m}$ Teufe tritt hier etwas Seeton auf.

\subsection{Archäologische Befunde}

Hinweise auf prähistorische Besiedelung sind von der Oberfläche des Beckens im Unterschied zu seinen Randgebieten nicht vorhanden. Jedoch wurde ein urnenfelderzeitliches Schwert einige Meter unter Saalach-Schotter in der St. Zeno-Terrasse, etwa 460 oder $455 \mathrm{~m}$ NN bei Froschham gefunden. Ein bronzezeitlicher Dolch wurde unterhalb Piding $(6 \mathrm{~km}$ nordöstlich des Kartenausschnittes) in $6 \mathrm{~m}$ Tiefe aus Saalach-Schottern geborgen und damit in rd. $445 \mathrm{~m}$ NN. Außerdem wurden im Saalach-Schotter und südlich St. Zeno römische Münzen geborgen (Menke 1971).

Zur Einstufung der obersten Schotter von REI 4 bringen die Gründung der Kirche St. Zeno im 8. Jh. n. Chr. Hinweise, ferner in Kirchennähe im Schotter in etwa $3 \mathrm{~m}$ Tiefe gefundene Gräber, die möglicherweise etwas älter als die Kirche sein können.

\subsection{Der bronzezeitliche Talboden}

Die voran gebrachten Angaben zeigen über die in REI 4 erfaßten feinkörnigen bis organogenen Sedimente, für die auch an anderen Stellen des Beckens Hinweise vorliegen, daß die Basis des bronzezeitlichen Talbodens etwa bei $450 \mathrm{~m}$ NN gelegen hat und daß im Bereich dieses Talbodens vorzugsweise feinkörnige bis organogene Sedimente abgesetzt wurden. Interessanterweise haben sich auch die Sole-Quellen auf dieses Niveau eingestellt. Damit zeigt sich ein beim Salinenbau gefundenes Bronzebeil (MENkE 1971), dessen Holzschaft von der Sole konserviert wurde und infolgedessen noch erhalten ist, in neuem Licht. Allerdings ist nicht mehr bekannt, in welcher Tiefe das Fundstück angetroffen wurde. Da jedoch die Sole in späteren Zeiten niemals obertägig austrat, sondern der Quellhorizont immer bei etwa $15 \mathrm{~m}$ unter dem heutigen Niveau gelegen haben muß, kann das Beil, das etwa ins 18. oder 17. Jh. v. Chr. zu datieren ist, nur aus dem Niveau des Quellhorizontes stammen.

Gegen Ausgang dieses Abschnittes hat die Schotterführung wieder zugenommen. So lagert in REI 4 ein Schotter bis in $4 \mathrm{~m}$ Tiefe auf. Ein weiterer Torf folgt darüber. Da der hangende Schotter ab $4 \mathrm{~m}$ Tiefe bis zur Oberfläche auf Grund seiner Einschlüsse nur römisch bis frühmittelalterlich sein kann, sollte der Torf in $4 \mathrm{~m}$ Tiefe, von dem keine Proben überliefert sind, etwa in die Eisenzeit bis Beginn der römischen Kaiserzeit gehören. Ein weiterer, höherer Talboden zeigt sich darin an.

Die unerwartet tiefe Lage des bronzezeitlichen Talbodens im Reichenhaller Becken kann nicht mit der Auslaugung des das Salz liefernden Haselgebirges in Zusammenhang gebracht werden. Abgesehen vom Niveau und der Richtung der Sole-Zulieferung sprechen auch die bronzezeitlichen Funde im Schotter unterhalb des Beckens gegen eine solche Möglichkeit. Bemerkenswert ist weiter, daß sich die heutigen Sole-Quellen im Bereich der Alten Saline von Bad Reichenhall an dieses bronzezeitliche Niveau des Talbodens halten und dies trotz erheblicher seitheriger Überschotterung.

\subsection{Oberflächennahe Bildungen westlich der Saalach}

Im Baugelände „Weitwiese“ war das folgende Profil vor Beginn der geologischen Untersuchung erschlossen. 
Profil „Baugrube Weitwiese“:

$\mathrm{Nr}$.

Karbonatgehalt

1 Gelbe, lehmige Schicht (unterhalb vom rezenten Hang)

$16,2 \%$

2 hellgraue, sandige Schicht in $\mathrm{rd} .120 \mathrm{~cm}$ Tiefe
3 hellgraue, schluffige Schicht in $130 \mathrm{~cm}$ Tiefe

$24,3 \%$

$24,4 \%$

- - - - - - -

4 schluffig-torfige Schicht in 140-145 cm Tiefe

$7,0 \%$

Schluff mit Torfbändchen in $145-155 \mathrm{~cm}$ Tiefe

$9,5 \%$

schluffig-torfige Schicht in 155-160 cm Tiefe

$38,0 \%$

Kleinkies, \pm zersetzt, mit grauem Schluff

n. b.

sandiger Kleinkies (Kalkschotter) in 160-180 cm Tiefe

n. b.

In den oberen Proben ( 1 bis 3 ) ist das Feinmaterial leicht dolomitisch ausgebildet. In Probe Nr. 6 scheint die Beteiligung von Seekreide für den etwas höheren Karbonatgehalt verantwortlich zu sein.

Geobotanik Profil „Baugrube Weitwiese“ (Tab. 2):

Die makrobotanische Untersuchung und die Pollenanalyse erbrachten die folgenden Ergebnisse:

Nr. 1: Ohne organische Beimengungen, nur mikroskopisch kleine, unfigurierte Humussteilchen.

Nr. 2: Ebenfalls ohne größere organische Beimengungen. Nach Kochen mit $\mathrm{KOH}$ zeigte sich eine schwache braune Färbung, die auf geringen Humusanteil schließen ließ.

Nr. 3: $130-140 \mathrm{~cm}$ schluffige Schicht mit Radizellen und Cyperaceenblattresten, einzelne Fragmente von Braunmoosen.

Nr. 4: $140-145 \mathrm{~cm}$ : schluffig-torfige Schicht mit Radizellen, Braunmoosresten (cf. Drepanocladus), 1 Same von Scirpus tabernaemontani.

Nr. 5: Schluff mit Torfbänder: zwischen $145-155 \mathrm{~cm}$ : Radizellentorf mit einzelnen Carex-Nüßchen.

Nr. 6: Schluffig-torfige Schicht bei $155-160 \mathrm{~cm}$ : Radizellentorf mit unbestimmbaren Holzresten. Rindenepidermis von Pinus.

Nr. 7: Ohne Inhalt.

Nr. 8: Ohne Inhalt.

Zur Pollenanalyse:

Nr. 2: Auf $3 \mathrm{~cm}^{2}$ : 1 Pollen von Picea, $1 \mathrm{cf}$. Salix, 1 Alnus, 2 Cyperaceen.

Nr. 3: Wenig Pollen, vermutlich selektiert. Auf $3 \mathrm{~cm}^{2}$ wurden gefunden: 7 Pinus, 24 Picea, 4 Alnus, 4 Betula, 2 Quercus, 5 Fagus, 6 Corylus, 14 Gramineen, 5 Cyperaceen und 7 indeterminata.

Nr. 4: Sehr hohe Picea-Werte, Fagus vertreten. Die Cyperaceenwerte deuten auf größere Cyperaceenbestände in der Nähe.

Nr. 5: Ahnlich wie Nr. 4.

Nr. 6: Koniferenwerte ähnlich wie Nr. 4, Fagus gleich hoch, EMW sehr niedrig. Getreide mit $0,6 \%$ vertreten, Chenopodiaceen als Kulturanzeiger.

Eine Aussage über das Alter der Proben zu machen, ist schwer möglich. Da die Buche vorhanden ist und in Nr. 6 Getreide erscheint, sind die untersten Proben nicht älter als jüngstes Subboreal, vermutlich subatlantisch. Die Spektren scheinen sehr stark lokal beeinflußt zu sein.

Dieser Befund wird durch die C14-Datierung eines Holzes aus einem benachbarten Kanalgraben präzisiert. An dieser zweiten Stelle blieb die geobotanische Untersuchung unergiebig.

$$
\begin{aligned}
& \text { Probe „Karlstein“ }- \text { Kn 1928: } 1900 \pm 50 \text { a BP } \\
& \text { (TK } 258243 / 44, \quad \text { R 45/63310, H 52/87150) }
\end{aligned}
$$


Tab. 2: Pollenspektren im Profil „Baugrube

Weitwiese" (Pollenzahl in \%)

\begin{tabular}{lrrc}
\hline & 4 & 5 & 6 \\
\hline Pinus & 19 & 27 & 28 \\
Picea & 52 & 50 & 44 \\
Abies & 12 & 10 & 10 \\
Betula & 3 & 1 & 0,6 \\
Quercus & 2 & 3 & 2 \\
Tilia & - & - & - \\
Ulmus & 3 & 1 & - \\
EMW & 5 & 4 & 2 \\
Alnus & 6 & 2 & 5 \\
Fagus & 3 & 5 & 5 \\
Corylus & 2 & 1 & 7 \\
Gramineen & 13 & 5 & 10 \\
Getreide & - & - & 0,6 \\
Cyperaceen & 84 & 67 & 35 \\
Plantago & 1 & 3 & - \\
Compositen & 4 & 2 & 5 \\
Chenopodiaceen & - & - & 0,6
\end{tabular}

Demnach gehört der hangende Lehm zeitlich etwa zu dem Schwemmfächer, der die obersten, römische Reste führenden Schotter der St. Zeno-Terrasse stellt. Vermutlich ist der unterlagernde Schotter etwa in den gleichen Zeitraum zu stellen; denn ein stärker ausgeprägter Boden ist nicht zwischengeschoben. Das schluffig-torfige Material scheint zusammengeschwemmt und die Anwitterung des liegenden Schotters kann viel jüngeren Datums, selbst nach Eindeckung mit Hochflut- oder Auelehm, erfolgt sein.

\subsection{Der römerzeitliche Talboden}

Damit zeichnet sich ein jüngerer, römischer Saalach-Schwemmfächer ab. Seine Hauptschüttung lag westlich der Saalach. Sie greift aber auch nach Osten über und bildet dort das oberste Lager der St. Zeno-Terrasse. In der westlichen Randzone wird die grobe Schüttung von feinkörnigen Ablagerungen teils abgelöst, teils überdeckt. Es ist sogar denkbar, daß gemäß den geringen Kalkgehalten des Lehms - Profil Weitwiese - die Zulieferung nicht überall durch die Saalach erfolgt ist, sondern beispielsweise als lokales Abspülmaterial dieser Zeit gedeutet werden muß.

\subsection{Jüngste Bildungen}

Jüngste Bildungen sind im Becken durch Seeablagerungen und durch Schotter in der Saalach-Aue vertreten.

\section{Saalach-See bei Karlstein:}

Die obersten Ablagerungen des ehemaligen Sees bestehen, soweit eine Aussage auf Grund einzelner, nicht tief reichender Bohrproben möglich ist, aus mehr oder weniger kalkhaltigem Ton, dem in verschiedenen Tiefen humose Schichten eingelagert sind (Profil Ka I/70 50-170 cm, Profil Ka WW/70 50-220 cm, beide Profile liegen in der Nähe der Grabungsstelle 1970). Die Bohrkerne zeigen die folgende Stratigraphie.

\section{Profil Ka I/70:}

$50-55 \mathrm{~cm} \quad$ Radizellentorf mit Braunmoos (Scorpidium)

$56-58 \mathrm{~cm}$ Übergang in humosen, kalkhaltigen Feinstsand, in dem einzelne stark humose Lagen eingebettet sind

bei $65 \mathrm{~cm}$ Übergang in kalkhaltigen, feinstsandigen Ton, Farbe graugrün mit einzelnen gelben Flecken 
$70-90 \mathrm{~cm}$ graugrüner, kalkhaltiger gelbfleckiger Ton

$100-120 \mathrm{~cm}$ stark humoser, stark kalkhaltiger, braungrauer Ton

$120-140 \mathrm{~cm} \quad 120-135 \mathrm{~cm}$ wie vorige Probe, zwischen $130-135 \mathrm{~cm}$ weniger kalkhaltig. 135$140 \mathrm{~cm}$ : stark toniger Humus, dunkelbraun

$150-160 \mathrm{~cm}$ humoser Schluff, hellbraungrau

$160-170 \mathrm{~cm}$ fast kalkfreier, sandiger, toniger Humus, dunkelbraun bis schwarz

\section{Profil Ka WW/70:}

$50-55 \mathrm{~cm} \quad$ gelbgrüner, kalkhaltiger Schluff

$55-68 \mathrm{~cm} \quad$ stark kalkhaltiger Torf mit Kalkknöllchen

$68-70 \mathrm{~cm}$ schwach kalkhaltiger, gelber Schluff

$100-112 \mathrm{~cm}$ stark humoser Ton, dunkelgraugrün

$112-116 \mathrm{~cm}$ tonige humose Schicht, dunkelbraun

$150-155 \mathrm{~cm}$ fast kalkfreier, humoser sandiger Ton, braun

$155-165 \mathrm{~cm}$ kalkhaltiger, stark humoser sandiger Ton, dunkelbraun

$165-170 \mathrm{~cm}$ brauner Schluff

200-210 cm Scorpidium-Torf (Braunmoostorf) mit Radizellen, mehr oder weniger tonig, kalkhaltig

210-218 cm gelbbrauner, humoser, fleckiger Ton bis Schluff

$218-220 \mathrm{~cm}$ graugrüner, schwach kalkhaltiger Ton

Tab. 3: Pollendiagramm im „Ehemaligen Saalach-See bei Karlstein“ (Pollenzahl in \%/.)

\begin{tabular}{|c|c|c|c|c|c|c|}
\hline Spektrum: I/70 & $\begin{array}{l}55 \\
\mathrm{~cm}\end{array}$ & $\begin{array}{l}120 \\
\mathrm{~cm}\end{array}$ & $\begin{array}{l}135 \\
\mathrm{~cm}\end{array}$ & $\begin{array}{l}160 \\
\mathrm{~cm}\end{array}$ & $\mathrm{WW} / 70$ & $\begin{array}{l}205 \\
\mathrm{~cm} \text { Tiefe }\end{array}$ \\
\hline Pinus & 42 & 11 & 17 & 11 & & 28 \\
\hline Picea & 27 & 6 & 5 & 2 & & 28 \\
\hline Abies & 7 & 6 & 1 & 5 & & 7 \\
\hline Betula & 1 & 6 & 10 & 11 & & 2 \\
\hline Quercus & 1 & 20 & 12 & 12 & & 5 \\
\hline Tilia & - & 1 & - & 1 & & 3 \\
\hline Ulmus & - & - & 1 & - & & 4 \\
\hline Fraxinus & - & 1 & - & 2 & & 1 \\
\hline EMW & 1 & 22 & 13 & 15 & & 13 \\
\hline Alnus & 6 & 19 & 31 & 24 & & 7 \\
\hline Fagus & 7 & 21 & 20 & 30 & & 13 \\
\hline Carpinus & - & 3 & 3 & 1 & & - \\
\hline Salix & - & 1 & 4 & 2 & & 5 \\
\hline Corylus & 3 & 12 & 32 & 20 & & 28 \\
\hline Juglans & - & 2 & - & - & & - \\
\hline Juniperus & - & 2 & - & 2 & & - \\
\hline Populus & 3 & 1 & - & - & & 2 \\
\hline Gramineen (Wildgrastyp) & 4 & 27 & 38 & 18 & & 20 \\
\hline Getreide Secale (Roggen) & 2 & 2 & 2 & 6 & & 3 \\
\hline Getreide Triticum (Weizen) & 3 & 2 & 1 & 2 & & 1 \\
\hline Plantago lanc. & 1 & 6 & 3 & 3 & & 3 \\
\hline Rumex & - & 1 & - & 2 & & 1 \\
\hline Chenopodiaceen & - & - & 1 & - & & - \\
\hline Compositen & 4 & 6 & 2 & 2 & & 9 \\
\hline Umbelliferen & - & 1 & 1 & - & & - \\
\hline Ericaceen & - & 2 & 3 & 1 & & - \\
\hline Charyophyllaceae & 2 & - & 2 & - & & 3 \\
\hline varia u. indeterminata & 4 & 18 & 26 & 17 & & 26 \\
\hline Cyperaceen & 20 & 3 & 17 & 7 & & 11 \\
\hline Flugasche & $\mathrm{x}$ & $\mathrm{xx}$ & $\mathrm{x}$ & $\mathrm{x}$ & & $\mathrm{x}$ \\
\hline
\end{tabular}


Die pollenanalytische Untersuchung (Tab. 3) einzelner Proben aus verschiedenen Tiefen zeigte, daß bis zu einer Tiefe von $205 \mathrm{~cm}$ die Buchenpollen anzutreffen sind und $\mathrm{z}$. T. sogar sehr hohe Werte aufweisen können. Eine genaue zeitliche Angabe erlauben die einzelnen Spektren leider nicht. In der Probe aus dem Scorpidium-Torf in $205 \mathrm{~cm}$ (Profil Ka $\mathrm{WW} / 70)$ ist die Buche mit $13 \%$ vertreten, wobei nicht feststeht, ob es sich um den Buchenanstieg oder nur um eine Depression der Buchenkurve handelt. Kulturspektren sind in allen Proben angetroffen worden. Sowohl Triticum-(Weizen) als auch Secale-(Roggen) Pollen wurden gefunden. Da Secale nördlich der Alpen erst durch die Römer eingeführt wurde (Rausch 1973, S. 65), sind die Ablagerungen in $205 \mathrm{~cm}$ Tiefe erst nach dem genannten Zeitpunkt erfolgt. Fast in allen Proben wurde Flugasche gefunden. Da sich Scorpidium-Torf, wie er in $205 \mathrm{~cm}$ Tiefe angetroffen wurde, nur im ganz seichten Wasser bilden kann, muß der Seespiegel z. Z. der Torfbildung ca. $200 \mathrm{~cm}$ tiefer gelegen haben als die heutige Oberfläche des ehemaligen Seebereiches.

Außer den Getreidepollen sind als Kulturanzeiger in den einzelnen Spektren noch vertreten: Plantago lanceolata (Spitzwegerich), Rumex (Sauerampfer), Chenopodiaceae (Beifußgewächse) und bei 120 und $135 \mathrm{~cm}$ Juglans (Walnuß). Dieser Pollen tritt frühestens in römischen, meist aber in frühmittelalterlichen Spektren auf.

Wieweit dieser ehemalige Saalach-See Bezug zu den limnischen Beckenablagerungen des älteren Holozäns (und eventuell auch des jüngeren Spätglazials) hatte, deren Obergrenze in $\mathrm{rd} .35 \mathrm{~m}$ Tiefe liegt, ist noch unbekannt.

\subsection{Junge Saalach-Schotter}

Im Auenbereich der Saalach, in welchen sich die Stadt Reichenhall inzwischen vorgeschoben hat, liegen jüngste Schotter, von denen nachfolgend ein Beispiel gebracht wird.

Neubau „Sparkasse“ gegenüber der Spielbank:

Mächtigkeit

$\mathrm{cm}$

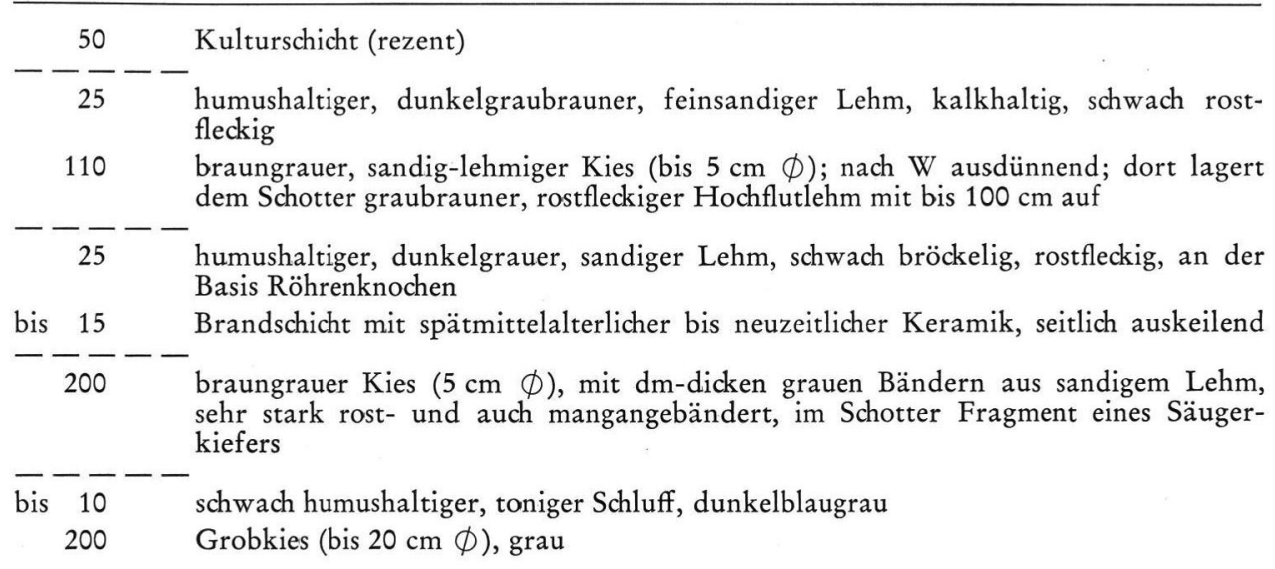

\section{Entwicklungsgeschichte}

Im jüngeren Atlantikum floß die mäandrierende Saalach auf der Froschhamer Terrasse. Ihre etwa niveaugleichen Mäander deuten auf eine nicht sehr große Wasserführung (Abb. 1). 
Gegen Ende des Atlantikums hat sich hier der Fluß um bald $20 \mathrm{~m}$ eingeschnitten. Dieser extreme Wert muß allerdings als Ergebnis der besonderen Gefällsverhältnisse im Innenraum des einstigen Saalach-Gletschers bewertet werden. Aufgegebene Mäanderbögen am Ausgang des Beckens füllen sich jetzt mit feinkörnigen bis torfigen Ablagerungen (Profil „Mittermüller"). Auch im Becken kam es im Subboreal zumindest abseits vom Flußlauf zur Sedimentation von Schluffen und zur Torfbildung.

Abschließend wurde, wie das bei Froschham in Kies eingebettete urnenfelderzeitliche Schwert zeigt, die Saalach-Aue um einige Meter aufgeschottert. Dafür kann die Zeit 3000 bis 3300 vor heute angesetzt werden.

In Zusammenhang mit in der Bronzezeit einsetzenden weitflächigen Rodungen wurden Materialverlagerungen in der Umgebung des Beckens (in diesem niederschlagsreichen Gebiet können sie nicht klimatisch bedingt sein) ausgelöst. Schwemmfächer legten sich damit auf trockengelegte Randbereiche des Beckens und des Saalach-Tales (z. B. „Mittermüller“, bis Marzoll zu verfolgen).

Ein breiter Schwemmfächer, der von der Saalach in das Becken hineingeschüttet wurde, läßt sich etwa in den Anfang unserer Zeitrechnung und in die anschließende Zeit einstufen. Auch in diesem Fall deuten sich Zusammenhänge mit der Besiedelung des BeckenRandes an. So lagern sich z. B. Auelehme als Abspülsedimente umgebender Böden ab.

Im Zusammenhang mit dieser weiteren Verfüllung des Beckens wurde der Fuß zuvor gebildeter in das Becken hineinlaufender lokaler Schwemmfächer überdeckt. Deswegen scheinen diese Schwemmfächer am Beckenrand geradezu abgeschnitten zu sein (Abb.1).

Die spätmittelalterliche bis neuzeitliche Entwicklung ist durch weitere, nicht sehr wirkungsvolle Umlagerungen im Auebereich gekennzeichnet, wie beispielsweise ein im Museum von Bad Reichenhall gezeigtes Gemälde (frühe Neuzeit?) andeutet.

\section{Regionale Beziehungen}

Im Ablauf des Holozäns ist hinsichtlich der Wasserführung unserer Flüsse in Mitteleuropa eine Generaltendenz erkennbar: Bis in die Bronzezeit hinein nimmt sie ab. Danach steigert sie sich wieder. Entsprechende Befunde liegen vom Eisernen Tor, aus dem Schweizer Mittelland und - wie neuerdings festgestellt - auch vom Niederrhein vor (BRUNNACKER 1971, 1974, z. T. unveröffentlicht). Die subborealen Feinsedimente im Reichenhaller Becken fügen sich ebenfalls in diesen Befund.

Jedoch wird diese Grundentwicklung durch fluviatile Aktivierungsphasen, die sich zumindest im jüngeren Holozän größenordnungsmäßig alle tausend Jahre wiederholen (BRUNNACKER 1960, 1974), überlagert. In diesen Abschnitten, die nach dendrochronologischen Befunden jeweils einige hundert Jahre anzudauern scheinen (BECKER 1971), arbeiten die Flüsse bereichsweise ihre Aue auf und schütten das aufgearbeitete Material schwemmkegelartig talabwärts vor.

Im Alpenvorland, z. B. an der Isar, aber auch andernorts sind folgende, generell als "Stufen" bezeichnete Phasen erfaßt (BRunNacker 1960):

Pulling-Stufe: Beginn rd. 4300 a BP

Lerchenfeld-Stufe: Beginn etwa 2. Jh. n. Chr.

Dazu kommt eine spätmittelalterliche bis frühneuzeitliche Stufe, die sich bislang präziserer Datierung entzogen hat.

Mit der Pulling-Stufe läßt sich zwanglos die erhebliche Eintiefung der Saalach am Ausgang des Beckens von Reichenhall als zeitlich entsprechender Ausräumungsbereich in Zusammenhang bringen. 
Bemerkenswert ist eine weitere Aktivierungsphase, die rd. 3000 bis 3500 Jahre zurückliegt. Sie zeigt sich über begrabene Baumstämme in den Tälern mancher Flußsysteme an. Im Alpenvorland war sie aber bislang kaum zu fassen. Erst neuerdings gibt es dafür einen Befund bei Weitwörth an der Salzach (Hell 1968), im Raum südlich von München einen ebensolchen bei Hechendorf am Pilsensee (Menke unveröff.), so daß neben dem beschriebenen Reichenhaller Becken sich jetzt auch hier die Funde zu mehren beginnen. Wenn das Reichenhaller Becken als Modell herangezogen wird, dann ist es denkbar, daß diese Phase deshalb nicht oder nur schwer über Oberflächenkartierungen zu fassen ist, weil damals die Talböden besonders tief gelegen haben und deswegen unter jüngeren Talböden begraben liegen. Erst neuerdings war es beispielsweise möglich, entsprechend alte Bildungen im Einflußbereich des Aare-Schwemmkegels und am Niederrhein zu fassen.

Die Lerchenfeld-Stufe ist im Becken durch den Schwemmfächer vertreten, der die obersten $4 \mathrm{~m}$ der St. Zeno-Terrasse aufbaut. Auch bei Epfach am Lech ist diese Stufe in das 2. Jh. n. Chr. zu stellen (BRunNACKer 1964), was nachfolgend wieder in Vergessenheit geraten ist (DIEz 1968). Zu ihr gehört ferner ein durch Holz mit $1505 \pm 65$ a BP datierter Isar-Schotter bei Landshut (Hofmann 1973). Dazu kommen neuerdings weitere Hinweise an der Aare und am Niederrhein, die an anderer Stelle behandelt werden sollen.

Zwanglos ist damit die Entwicklung des Reichenhaller Beckens in die Gesamtentwicklung der Flüsse am Alpenrand und darüber hinaus in Mitteleuropa eingefügt. Doch sind die Ursachen sowohl für die generelle wie die spezielle Entwicklung noch nicht bekannt. Neben klimatischen Gegebenheiten, die übrigens gar nicht sonderlich ausgeprägt gewesen sein müssen, spielen zumindest an der Saalach wesentliche anthropogene Eingriffe eine gewisse Rolle, wenigstens hinsichtlich der Auslösung von Bodenerosion in der Umgebung.

Wie diese Vorgänge ineinander gewirkt haben, ist ebenfalls offen. Bemerkenswert ist aber immerhin, daß bereits in der späten Bronzezeit die Zebrina detrita (MülLER) aus dem mediterranen Bereich eingewandert ist (M. BRunNACKeR in K. BrunNACKeR 1970). Sie benötigt in offenem Gelände warme und lichte Standorte mit nicht zu tonigen Böden (M. \& K. BrunNacker 1959). Unbekannt ist, ob allein die Rodungen ausgereicht haben für das Einwandern dieser Schnecke. Schließlich folgen erst in der Eisenzeit die Helicellen als Mollusken mit ähnlichen, letzten Endes aber doch nicht ganz so ausgeprägten Ansprüchen.

\section{Schriftenverzeichnis}

BECKER, B. (1971): Zwischenbericht über die dendrochronologische Bearbeitung subfossiler Eichen aus Flußterrassenschottern des südlichen Mitteleuropa. - Jb. Akad. Wiss. u. Lit. f.d.J. 1971: 140-145, 2 Abb.; Mainz.

Brunnacker, K. (1960): Zur Kenntnis des Spät- und Postglazials in Bayern. - Geol. Bavarica 43: 74-150, 13 Abb., 16 Tab.; München.

- (1964): Die geologisch-bodenkundlichen Verhältnisse in Epfach. - Studien zu AbodiacumEpfach: $140-156,3$ Abb., 1 Tab., Tafel 56-57 sowie 1 Textbeilage; München.

- (1967): Geologisch-bodenkundliche Beobachtungen in der neolithischen Station von Burgäschisee-Süd. - Acta Bernensia II: Seeberg - Burgäschisee-Süd, Teil 4 (Chronologie und Umwelt): 141-143; Bern.

- (1970): Spät- bis postglaziale Höhlensedimente in der Schwäbischen Alb. - Manuskript (erscheint in der Monographie von TAUTE über das Mesolithikum Süddeutschlands).

- (1971): Geologisch-pedologische Untersuchungen in Lepenski Vir am Eisernen Tor. - Fundamenta A 3: 20-32, $6 \mathrm{Abb}$; Köln/Wien.

(1974): Bemerkungen zur holozänen Flußentwicklung. - Heidelberger Geogr. Arb. 40: 239248, 3 Abb.; Heidelberg.

Brunnacker, M. \& Brunnacker, K. (1959): Gehäuseschneckenfauna und Boden. - Zoolog. Anz. 163: 128-134, 4 Tab.; Leipzig.

Diez, Th. (1968): Die würm- und postglazialen Terrassen des Lech und ihre Bodenbildungen. Eiszeitalter u. Gegenwart 19: 102-128, 6 Abb., 6 Tab.; Öhringen (Rau). 
Firbas, F. (1923): Pollenanalytische Untersuchungen einiger Moore der Ostalpen. - Lotos 71: 187-242; Prag.

Hell, M. (1968): Steinkeule und Ringbarren als Flußfunde aus der Salzach. - Archaeologia Austriaca 43: 121-124; Wien.

Herm, D. (1962): Die Schichten der Oberkreide (Untere, Mittlere und Obere Gosau) im Becken von Reichenhall (Bayerische/Salzburger Alpen). - Z. dt. geol. Ges. 113: 320-338, 4 Abb.; München.

Hillebrandt, A. von (1962): Das Alttertiär im Becken von Reichenhall und Salzburg (Nördliche Kalkalpen). - Z. dt. geol. Ges. 113: 339-358, 7 Abb.; Hannover.

Heuberger, H. (1972): Die Salzburger "Friedhofterrasse“ - Eine Schlernterrasse? - Z. Gletscherkde. u. Glazialgeol. 8: 237-251, 3 Abb.; Innsbruck.

Hofmann, B. C. (1973): Geolog. Karte von Bayern $1: 25000$ Nr. 7439 Landshut Ost mit Erläut., 113 S., 7 Abb., 12 Tab., 7 Taf. u. 2 Beil.; München.

Janik, Ch. V. (1969): Die Pfahlbausiedlung See/Mondsee im Blickfeld landschaftlicher Forschung. - Jb. Oberösterreich. Musealver. 114: 181-200, 3 Karten, 2 Schnitte, Taf. XIII u. XIV; Linz.

Krauss, H. (1913): Geologische Aufnahme des Gebietes zwischen Reichenhall und Melleck. Geognost. Jh. 26: 105-154, 2 Taf., 1 geol. Karte; München.

Lürzer, E. (1956): Die postglaziale Waldgeschichte des Salzburger Vorlandes. - Mitt. Ges. f. Salzburger Landeskde. 96: 223-234; Salzburg.

MaYeR, H. (1966): Waldgeschichte des Berchtesgadner Landes (Salzburger Kalkalpen). - Forstwiss. Forsch. Beih. z. Forstwiss. Cbl. 22: 1-42; Hamburg/Berlin.

Menke, M. (1968): Schrötlingsformen für keltisches Silbergeld aus Karlstein, Ldkr. Berchtesgaden (Oberbayern). - Germania, 46: 27-35, 1 Beil.; Frankfurt a. M.

- (1971): Vor- und frühgeschichtliche Topographie in Karlstein und Bad Reichenhall. - Führer vor- u. frühgesch. Denkmäler, 19: 140-160; Kallmünz.

- (1973): Eine gallische Potin-Münze aus Karlstein bei Bad Reichenhall. - Jb. Numismatik u. Geldgesch. 23: 89-91, 1 Abb., 7 Taf.; Kallmünz.

Paul, H. \& RuofF, S. (1927): Pollenstatistische und stratigraphische Mooruntersuchungen im südlichen Bayern. I. - Ber. Bayer. Bot. Ges. 19: 1-84; München.

RaUSCH, K. A. (1973): Untersuchungen zur spät- und nacheiszeitlichen Vegetationsgeschichte im Gebiet des ehemaligen Inn/Chiemseegletschers. - Diss. Univ. Göttingen.

Schirmer, W. (1973): The Holocene of the Former Periglacial Areas. - Eiszeitalter u. Gegenwart 23/24: 306-320; Öhringen (Rau).

Schmeide, H. (1971): Ein Beitrag zur spätglazialen Vegetations- und Waldentwicklung im westlichen Salzachgletschergebiet. - Eiszeitalter u. Gegenwart 22: 110-126, 5 Abb.; Öhringen (Rau).

- (1973): Zur Vegetations- und Waldentwicklung im Frillenseegebiet. - Erl. Geol. Kt. von Bayern 1: 25 000, Nr. 8242 Inzell: 74-80, 2 Abb.; München.

Manuskr. eingeg. 10. 11. 1975. 